\title{
A Case of Unresectable Pancreatic Cancer with Long-Term Survival in High-Intensity Focused Ultrasound (HIFU) Therapy
}

\section{Introduction}

Despite advancements in imaging diagnosis, about $60 \%$ of cases of pancreatic cancer (PC) are still unresectable. Chemotherapy and radiochemotherapy are commonly performed as alternative treatments, but satisfaction with the results remains low. High intensity focused ultrasound therapy (HIFU) is expected to be a minimally invasive therapy for these cases. HIFU is an epoch-making therapeutic method and is a local therapy that converges a large number of ultrasonic transmission sources at one point at the target site of the tumor and cauterizes tissues from outside the body [1-3].

\section{Case Description}

A 63-year-old male, who underwent CT examination at another medical center due to epigastric pain, was then diagnosed with a low-density tumor $(65 \mathrm{~mm})$ infiltrating the celiac artery (CA) and superior mesenteric artery (SMA) in the pancreas body ( $\mathbf{F i g}$. 1a). It was histologically diagnosed as PC (UICC criteria; stage III) by EUS-FNA. There was no suitable surgery. Therefore, chemoradiotherapy was initiated due to locally advanced PC. Chemotherapy was performed with GS therapy (GEM $1000 \mathrm{mg} / \mathrm{m}^{2}+\mathrm{S}-1100 \mathrm{mg} /$ day), and radiotherapy with $50.4 \mathrm{~Gy} / 28$ fraction. After receiving GS therapy (GEM $1000 \mathrm{mg} /$ $\mathrm{m}^{2}+\mathrm{S}-1100 \mathrm{mg} /$ day) for 2 months, the PC was still diagnosed as progressive disease
(PD) based on WHO criteria by CT. The patient presented at our hospital for HIFU therapy. Under sufficient informed consent, HIFU therapy was performed as an additive therapy to systemic chemotherapy. This clinical trial was approved by the ethics committee of our hospital (IRB: 890) and registered with University Hospital Medical Information (UMIN) and was initiated (UMIN000009969). B-mode US showed a hypoechoic mass in the pancreas body ( Fig. 2a), and contrast-enhanced ultrasound (CE-US) showed isovascularity in previous HIFU therapy ( $>$ Fig. 2b). The FEP-BY 02 HIFU Therapeutic System was used as the HIFU device. (Yuande Bio-Medical Engineering, Beijing, China) (๖ Fig. 3).

After fasting for $12 \mathrm{~h}$ before therapy, the patient was placed in a supine position and observed with B-mode ultrasound in order to determine the appropriate therapeutic route and the relation with surrounding organs before therapy. Low-concentration ultrasonic gel for HIFU was applied to the therapeutic area, deaerated water was placed in the water sac, the transducer was lowered to the therapeutic area, and the target was observed with the image confirmation probe while slight compression was applied with the water sac. The image confirmation probe and the therapeutic oscillator were set on the same axis and a therapeutic plan was formulated while watching the target. The thickness of the abdominal wall (skin, subcutaneous fat and muscles of the abdominal wall), the distance from the skin to the target, and the size and depth of the tumor were measured. The therapeutic dose was then calculated considering the attenuation due to the tissue and surrounding environment. The therapeutic plan, output power and HIFU therapy position were entered into the computer. The range cauterized by one irradiation was as small as $3 \times 3 \times 10 \mathrm{~mm}$. Therapy was carried out while confirming the US image in real time while moving $3 \mathrm{~mm}$ vertically and $3 \mathrm{~mm}$ to the left and right. During the operation, no significant pain was registered. Therapy was set as one set of 2-3 treatments every 3 days, with the treatment time averaging $30 \mathrm{~min}$. The target HIFU irradiation was 1000 per dose.

The clinical course is shown in > Fig. 4 The tumor showed echogenic change immediately after HIFU therapy on B-mode US ( - Fig. 2c) and a hypovascularity change on CE-US ( $>$ Fig. 2d). The CT image taken after 3 months of HIFU therapy also showed that the tumor had shrunk ( $>$ Fig. 1b). As for symptoms, the amount of pain had been significantly reduced. Therefore, the amount of opioid prescribed was also reduced. Additional HIFU therapy (2nd) was administered in order to obtain further therapeutic results. As seen on the CT image taken 5 months after the first therapy, the tumor was further reduced and PR as the thera-
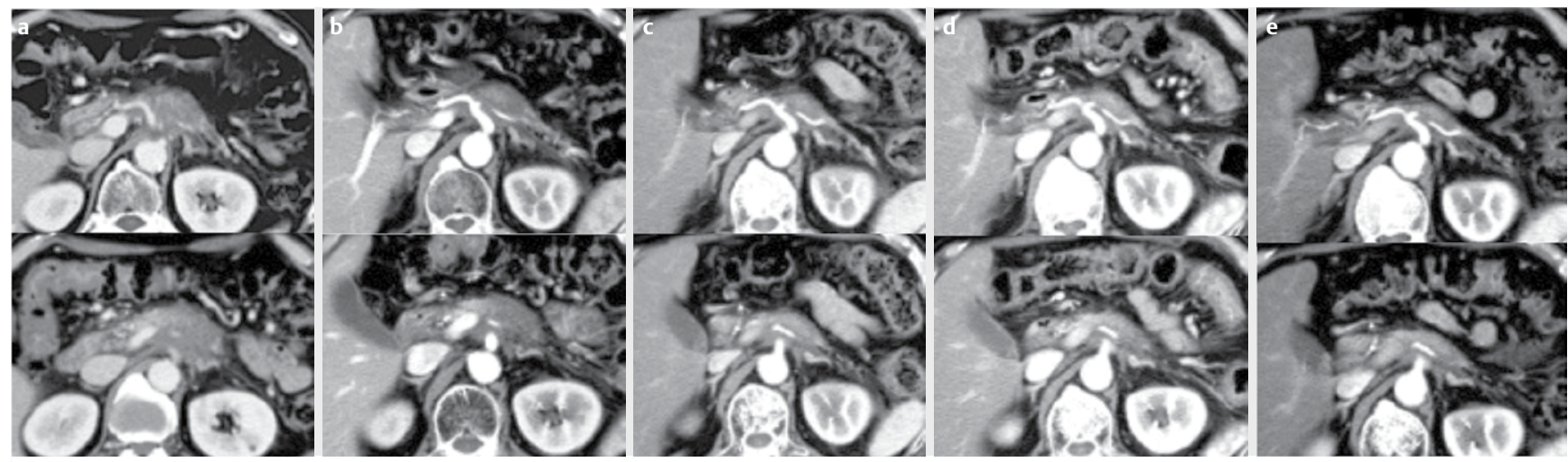

- Fig. 1 CT imaging: a: CT before HIFU therapy showed a low-density mass $(65 \mathrm{~mm})$ infiltrating the CA and SMA in the pancreas body. b: CT imaging 3 months after HIFU therapy showed that the pancreas tumor had shrunk slightly. c: CT imaging after the second HIFU therapy (5 months after the first HIFU therapy) showed that the tumor had shrunk and PR in the therapeutic effect was obtained. d: CT imaging after the 4th HIFU therapy (19 months after the first HIFU therapy) showed the tumor size was unchanged (PR continued). e: CT imaging after the 7th HIFU therapy (39 months after the first HIFU therapy) showed continued PR until the patient passed away. 

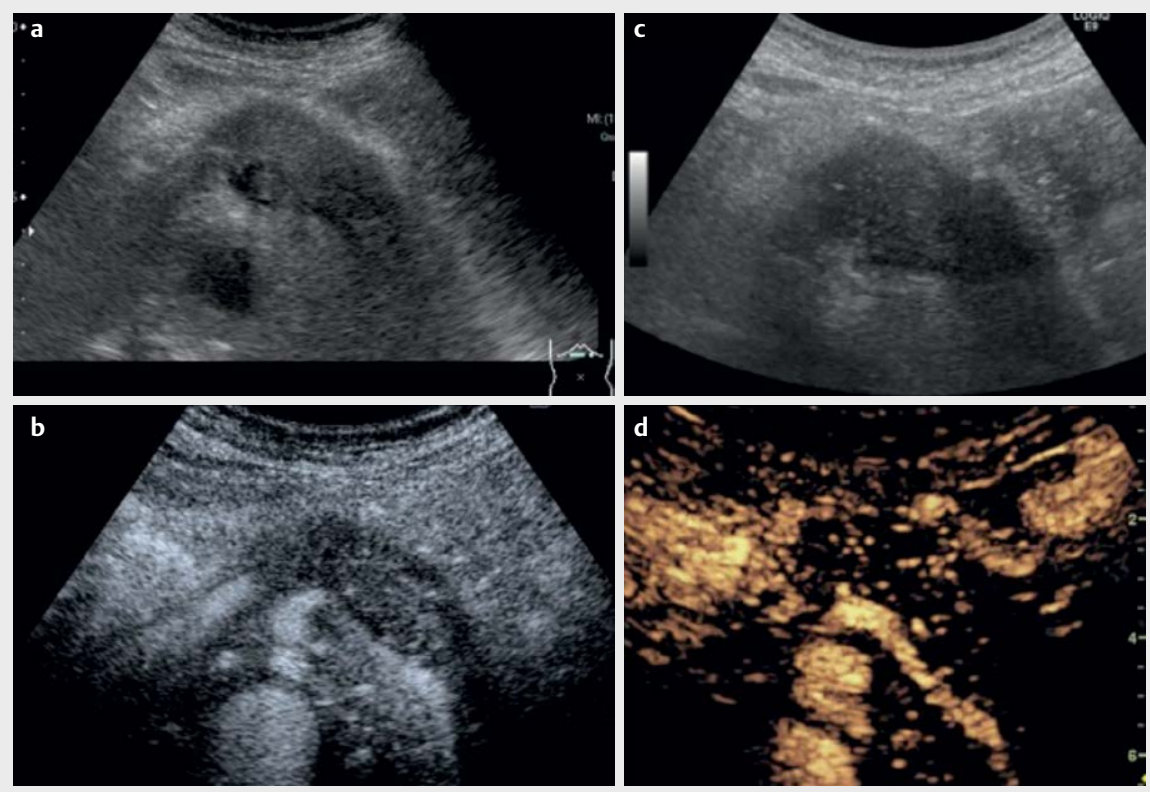

- Fig. 2 US imaging: a: B-mode US before HIFU therapy showed a hypoechoic mass ( $65 \mathrm{~mm}$ ) in the pancreas body. b: CE-US before HIFU therapy showed an isovascular mass in the pancreas body. c: B-mode US after HIFU therapy showed echogenic change in the tumor post-HIFU therapy. d: CE-US after HIFU therapy showed a change in vascularity from isovascularity to hypovascularity

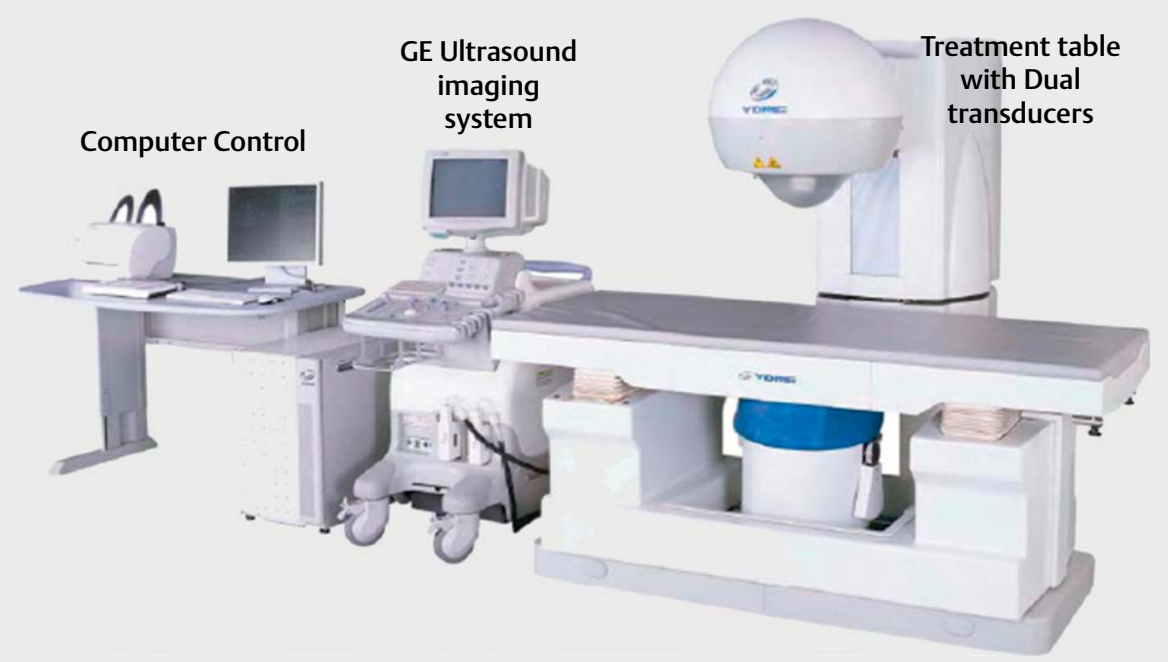

- Fig. 3 FEP-BY Series HIFU Therapy System: The system includes computer control, GE ultrasound imaging system, and treatment table with dual transducers.

peutic effect was also obtained ( $>$ Fig.1c). Because of increased pain, additional HIFU therapy (3rd treatment) was administered. On the CT image taken 19 months after the first HIFU treatment, the tumor marker was elevated and epigastric discomfort had increased. Since the tumor size was unchanged, stable disease (SD) ( $\triangleright$ Fig.1d), additional HIFU therapy (4th therapy) was performed. As seen on the CT image taken
26 months after the first HIFU therapy, the tumor size was unchanged and the patient's pain had improved. However, tumor marker elevation was observed, and an additional HIFU therapy ( $5^{\text {th }}$ therapy) was performed. Even after that, no marked change was noted in the tumor size on the image. However, since the tumor marker temporarily decreased and then increased again, two additional HIFU treatments were performed (34 and 39 months after the first HIFU therapy). Regarding chemotherapy, GS therapy also continued until the 6th HIFU therapy and then changed to GEM + nab-PTX therapy 3 years and 6 months after the introduction of chemotherapy. However, although there was no change on the image ( $\triangleright$ Fig. 1e), the general condi- 


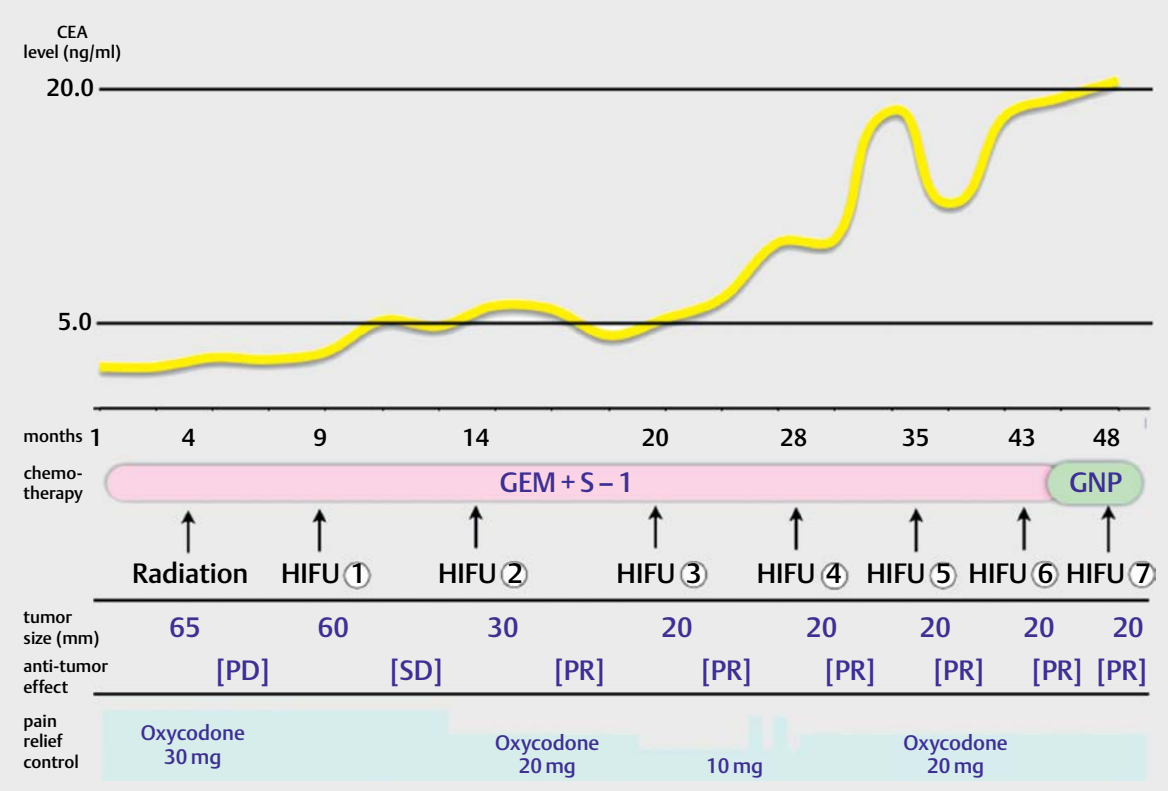

- Fig. 4 Clinical course; each treatment, tumor size, tumor marker (CEA label), anti-tumor effect, pain relief control were showed in the monthly time series as the clinical course.

tions, such as the appearance of edema, had worsened and the malaise had increased. Ultimately, the patient died 4 years and 6 months after the onset of $P C$ and 3 years and 6 months after the start of HIFU therapy after being diagnosed with PD.

\section{Discussion}

HIFU therapy which can be performed repeatedly over a long period of time with $\mathrm{mi}$ nimal invasiveness is expected to be one of the effective therapies for locally advanced PC $[4,5]$. HIFU therapy depends on the thermal action (thermal energy; only the focal area increases to 80-100 degrees) and the non-thermal action (mainly cavitation), which is the biological action of ultrasonic waves. It does not affect tissue outside of the focal region. HIFU therapy causes coagulation necrosis, degeneration/apoptosis, cell destruction, and tissue fibrosis, and has a therapeutic effect [1-3]. It has been suggested that HIFU therapy results in the enhancement of drug permeation and cancer-specific immunity due to tumor collapse. It is anticipated that an antitumor effect will be obtained, and it is expected to have many potential benefits such as the possibility of retreatment/additional therapy, prolongation of survival time, pain alleviation effect by blocking nerves.
Indication organs in both benign and malignant cases are the liver, pancreas, pelvis (urinary bladder tumors, uterine fibrosis, prostate tumors, prostate hypertrophy, rectal tumors), kidney, breast, extremities, bone, lymph node, retroperitoneum, and brain. HIFU is contraindicated in the digestive tract, gall bladder, and biliary tract in order to avoid the risk of perforation and leakage. The anticipated adverse events from HIFU therapy were skin burn, pancreatitis, pancreatic pseudocyst, leakage of pancreatic and bile duct juice, intestinal perforation, gastrointestinal bleeding, tumor bleeding, hematoma, obstruction of the celiac and superior mesenteric arteries, and tumor dissemination [1, 2].

In this case PD was diagnosed after chemoradiation therapy with GS therapy. Chemotherapy with GS therapy was continued without change until the 6th HIFU therapy was completed. This also indicates that the tumor reduction effect after PD in this case can be judged to be the enhancement of the therapeutic effect due to the drug permeation effect caused by HIFU therapy. Since HIFU therapy is different from radiotherapy, it is possible to treat repeatedly as many times as necessary. The ability to treat over a long period is also beneficial. HIFU therapy may be one of the strategies for locally advanced unresectable PC, and an antitumor effect and pain-relieving effect can be expected. HIFU therapy in combination with chemotherapy as systemic therapy has the potential to contribute to the prolonged survival of PC patients. Further studies, such as the enhancement of the therapeutic effect due to the drug penetration effect, the possibility of surgical therapy, preoperative therapy for borderline resectable $P C$, and the possibility for drug/delivery system therapy, are expected in the future.

Nevertheless, HIFU therapy has some limitations. It uses ultrasonography and treatment can be performed in real time and in a short time, but it is thought to be not objective. Additionally, the method is operator-dependent. Finally, randomized controlled trials should be conducted to determine whether HIFU therapy could be useful as a palliative PC therapy in the future.

Supported by the Tokyo Medical University Cancer Research Foundation.

\section{Conflict of Interest}

The authors declare that they have no conflict of interest. 


\section{Authors}

Atsushi Sofuni, Mitsuru Fujita, Yasutsugu Asai, Takayoshi Tsuchiya, Kentaro Ishii, Reina Tanaka, Ryosuke Tonozuka, Mitsuyoshi Honjo, Shuntaro Mukai, Kazumasa Nagai, Kenjiro Yamamoto, Yukitoshi Matsunami, Takashi Kurosawa, Hiroyuki Kojima, Takao Itoi

\section{Affiliation}

Department of Gastroenterology and Hepatology, Tokyo, Japan

\section{Prof. Atsushi Sofuni}

Depat of Gastroenterology and

Hepatology

6-7-1 Nishisinjuku

Shinjuku-ku Tokyo

Tokyo

160-0023

Japan

Tel : + 81-3-3342-6111

a-sofuni@amy.hi-ho.ne.jp

Bibliography

DOI https://doi.org/10.1055/a-1061-7790

Ultrasound Int Open 2019; 5: E89-E92

(c) Georg Thieme Verlag KG Stuttgart .

New York

ISSN 2199-7152

()⑴ $(8)$
References

[1] He S-X, Xiong L-L, Yao S-S et al. The preclinical research of high intensity focused ultrasound. J Beijing Med Univ 1999; 31: 573-576

[2] Dubinsky T], Cuevas C, Dighe MK et al. High-intensity focused ultrasound: current potential and oncologic applications. AJR 2008; 190: 191-199

[3] Sofuni A, Moriyasu F, Itoi T et al The current potential of high-intensity focused ultrasound for pancreatic carcinoma. J Hepatobiliary Pancreat Sci 2011; 18: 295-303

[4] Sofuni A, Moriyasu F, Itoi T et al. Safety trial of high-intensity focused ultrasound therapy for pancreatic cancer. World J Gastroenterol 2014; 20: 957

[5] Sofuni A, Moriyasu F, Itoi T et al. Highintensity focused ultrasound therapy for pancreatic cancer. Jpn J Med Urtasonics 2014; 41: 727-733 